\title{
Service Evaluation Audit of Awake Craniotomy Cases in a UK Tertiary Referral Teaching Hospital
}

\section{Liew G., ${ }^{1}$ Prakash A., ${ }^{2}$ Adapa R., ${ }^{2}$ Santarius T., ${ }^{2}$ Duane D. ${ }^{2}$ \\ ${ }_{1}$ Singapore General Hospital, Outram Rd, 169608 Singapore. \\ ${ }^{2}$ Addenbrooke's Hospital, Hills Road, Cambridge, CB20QQ UK.}

\section{BACKGROUND: Awake craniotomy (AC)} procedures are commonly performed for epilepsy and deep brain stimulation surgery and to help preserve eloquent areas during brain tumour resection. While awake procedures for craniotomy surgery are increasingly being used to reduce the length of hospital stay, intensive care admissions and avoid the risks of general anaesthesia (GA), they can pose a unique challenge to neuroanaesthetists. ${ }^{1}$ The aim of our audit was to review the current anaesthetic practice and patient outcomes for awake surgery in our tertiary referral teaching centre neurosurgical unit at Addenbrooke's Hospital, Cambridge, UK.

METHODS: This service evaluation audit did not require ethics committee approval. We retrospectively obtained a database of all patients who underwent awake craniotomy neurosurgery from $1 / 1 / 15$ to $15 / 5 / 17$ from the hospital's information technology (IT) department. Using Microsoft Excel( ) (Microsoft Corporation USA) for data entry and analysis, the required information was collected from the electronic anaesthetic and surgical records.

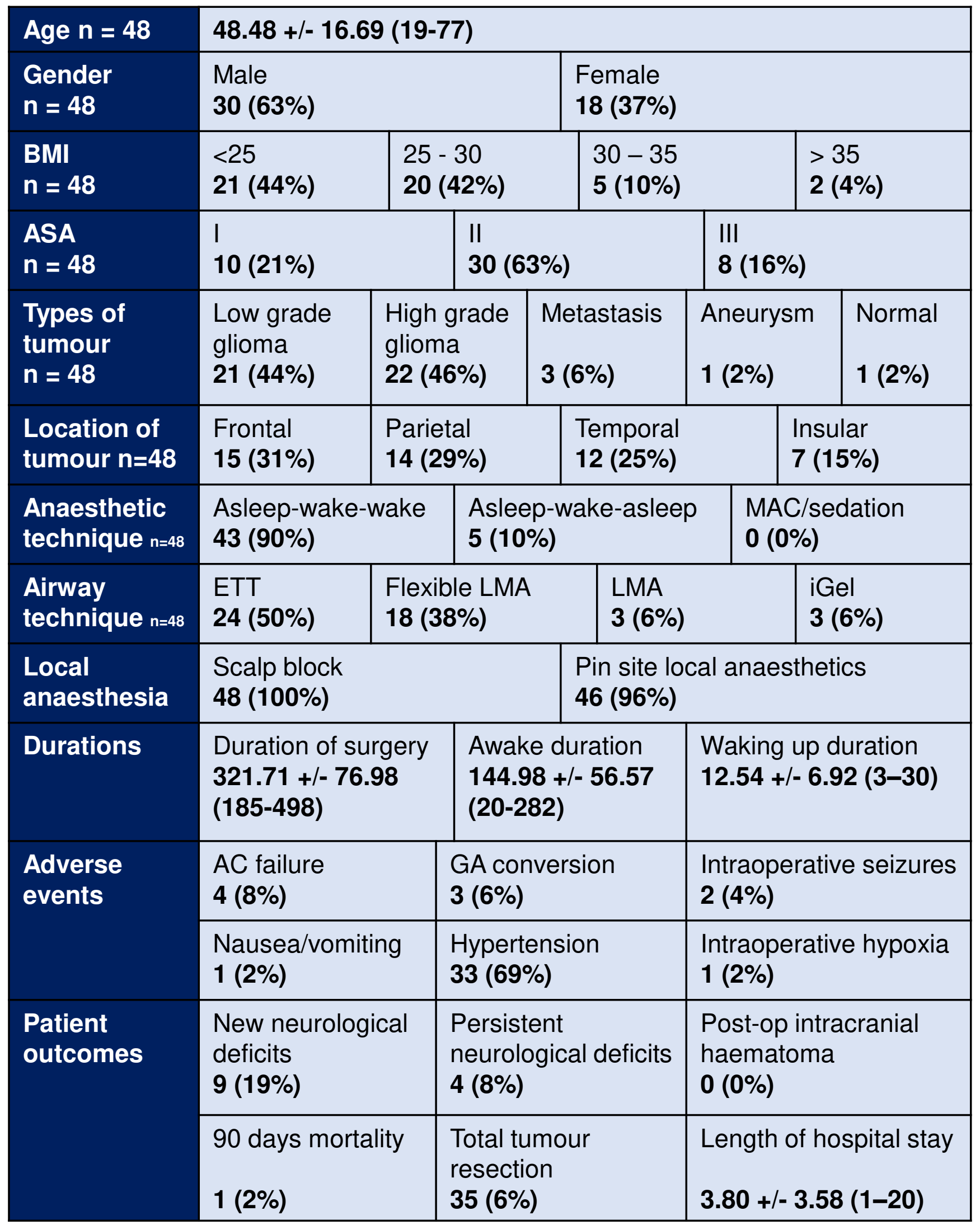

RESULTS: 510 patients underwent craniotomy surgery over $2 \frac{1}{2}$ years and 48 cases were done as awake procedures. 13 patients were listed for awake procedures but had the operation done under GA due to surgical difficulties (7), anaesthetic concerns (2) and awake technique not deemed necessary (4). The preoperative airway assessment of most patients was without concern and only one patient was predicted difficult. With regards to the anaesthetic techniques used for the awake surgery, 43 patients were asleep-wake 5 patients were asleep-wake-asleep (AWA) and no patients had local anaesthesia alone with or without conscious sedation. Three of the AWA patients were unplanned due to a combination of agitation/brain herniation; or a decrease in their Glasgow Coma Score (GCS). All of these patients had a laryngeal mask airway (LMA - Intersurgical Ltd UK) reinserted for conversion to GA. The two planned AWA patients had their airway secured with an endotracheal tube (ETT) reinserted using an AirTraq $\odot$ video laryngoscopy device (Prodol Meditec). In all cases, the airway techniques used for the asleep phase of the procedure was evenly divided into an ETT (24) and a LMA (24). Three patients who initially had a LMA inserted had to be subsequently intubated due to ventilation problems while one patient who had an ETT placed had severe coughing on awakening. Two patients had seizures during their awake phase - the first resolved spontaneously while the second had recurrent seizures despite levetiracetam, phenytoin and brain irrigation with cold saline). In total, there were 4 cases $(8 \%)$ where awake surgery ultimately failed due to one case of intraoperative recurrent seizures and three cases of unplanned AWA due to agitation and fluctuating GCS.

CONCLUSION: Our current practice of anaesthesia for awake craniotomy procedures is associated with a high degree of success for the patients. Despite the slightly higher GA conversion rate $(6 \%)$ and $A C$ failure rate $(8 \%)$ associated with our techniques compared with the reported $2 \%$ incidence for both for the outcomes in the literature, we cite our low sample size (48 patients) to account for this. ${ }^{2}$ As our case numbers and experience with these procedures increase we expect this rate to fall and patient outcomes to improve.

\section{References:}

1. Au K, Bharadwaj S, Venkatraghavan L, Bernstein M. Outpatient brain tumor craniotomy under general anesthesia. J Neurosurg 2016;125:1130-5

2. Stevanovic A, Rossaint $R$, Veldeman $M$, Bilotta $F$, Coburn $M$. Anaesthesia management for awake craniotomy: Systematic review and meta-analysis. PLoS One 2016;11:e0156448 